\title{
ISOSCATTERING DEFORMATIONS FOR COMPLETE MANIFOLDS OF NEGATIVE CURVATURE
}

\author{
PETER PERRY AND DOROTHEE SCHUETH
}

\begin{abstract}
We construct continuous families of non-isometric metrics on simply connected manifolds of dimension $n \geq 9$ which have the same scattering phase, the same resolvent resonances, and strictly negative sectional curvatures. This situation contrasts sharply with the case of compact manifolds of negative curvature, where Guillemin/Kazhdan, Min-Oo, and Croke/Sharafutdinov showed that there are no nontrivial isospectral deformations of such metrics.
\end{abstract}

\section{Contents}

1. Introduction 1

2. Scattering Theory 3

2.1. The Spectral Shift 3

2.2. Resolvent Resonances 5

3. Isoscattering via Effective Torus Actions $\quad 7$

4. Applications 11

5. Non-Isometry 14

$\begin{array}{ll}\text { References } & 16\end{array}$

\section{INTRODUCTION}

Compact closed manifolds $X$ of strictly negative sectional curvature are infinitesimally spectrally rigid in the sense that any continuous isospectral deformation of the metric takes place in a single isometry class. The purpose of this paper is to show that, by contrast, there are continuous families of non-isometric metrics on non-compact manifolds which have strictly negative sectional curvatures and the "same spectrum" in a sense which we will make precise.

Our examples are constructed from conformally compact, asymptotically hyperbolic manifolds; we give the precise definition in section 2 of what follows. Such manifolds have at most finitely many $L^{2}$-eigenvalues and nonempty continuous spectrum with no embedded eigenvalues.

There are two natural notions of "spectral" data carried by the continuous spectrum. The first is the spectral shift, a real-valued function on $\mathbb{R}$ which is the analogue of the counting function for the eigenvalues of the Laplacian on a compact manifold.

Key words and phrases. Geometric scattering, isophasal manifold.

2000 Mathematics Subject Classification. 58J53, 58J50.

The first author was supported in part by NSF grant DMS-0100829 and DMS-0408419.

The second author was supported in part by the DFG Priority Programme 1154. 
The spectral shift is defined with respect to a background metric $g_{0}$ which renormalizes the problem (see section 2.1). A celebrated formula of Birman and Krein shows that the spectral shift is identical to the scattering phase. A second analogue of "spectral" data for the Laplacian on an asymptotically hyperbolic manifold is the set of eigenvalues and resolvent resonances, enumerated with multiplicity. Resolvent resonances are poles of Green's function for the Laplacian, meromorphically continued from its initial domain of definition on a cut plane to covering surface (see section 2.2).

If $\left(X, g_{0}\right)$ is a complete, non-compact Riemannian manifold, two complete metrics $g_{1}$ and $g_{2}$ on $X$ are said to be isophasal if the spectral shift functions for the pairs $\left(\Delta_{1}, \Delta_{0}\right)$ and $\left(\Delta_{2}, \Delta_{0}\right)$ are equal almost everywhere, and isopolar if the eigenvalues and resolvent resonances of $\Delta_{1}$ and $\Delta_{2}$ coincide. If $g_{1}$ and $g_{2}$ are both isopolar and isophasal, we will say that these metrics are isoscattering. We will develop sufficient criteria for two metrics to be isoscattering; our main class of examples are isoscattering metrics on $\mathbb{R}^{n}$ :

Theorem 1.1. Suppose that $g_{0}$ is a complete metric on $\mathbb{R}^{n \geq 9}$ which is invariant under the canonical $O(n)$-action, and let $K$ be any $O(n)$-invariant compact subset of $\mathbb{R}^{n}$. There exist continuous families of complete, non-isometric metrics $\left\{g_{\alpha}\right\}$ on $X$ so that the $g_{\alpha}$ are mutually isophasal. Moreover, the metrics $g_{\alpha}$ coincide with $g_{0}$ on $\mathbb{R}^{n} \backslash K$ and may be chosen arbitrarily close to $g_{0}$ in the $\mathcal{C}^{\infty}$ topology on symmetric two-tensors. If $g_{0}$ is asymptotically hyperbolic, then the metrics $\left\{g_{\alpha}\right\}$ are also isopolar.

Note that the $g_{\alpha}$ are claimed to be isophasal/isopolar to each other (not to $g_{0}$ ).

Remark 1.2. Letting $m=n-4$, the parameter space for the continuous families of isophasal metrics on $\mathbb{R}^{n}$ that we construct has dimension

$$
d \geq \frac{m(m-1)}{2}-\left[\frac{m}{2}\right]\left(\left[\frac{m}{2}\right]+2\right)>1
$$

if $n=9$ or $n \geq 11$. (If $n=10$, the parameter space has dimension at least 1 ).

Remark 1.3. (i) The condition that $g_{0}$ be asymptotically hyperbolic is only used to ensure that the resolvents of the operators $\Delta_{\alpha}$ can be mermorphically continued, so that resolvent resonances are well-defined. Presumably, isopolar metrics can also be constructed for other classes of metrics in geometric scattering theory (such as those discussed in Remark 4.10) although we have not done so here.

(ii) Theorem 1.1 will follow from a specific application (see Remark 4.8(ii)) of some general isophasality/isopolarity principles (Theorems 3.2/3.3, successively specialized in Theorems 4.3 and 4.7), and from a nonisometry proof given for this specific class of metrics in section 5 (see Theorem 5.1).

From Theorem 1.1 and the fact that the sectional curvatures of a metric of negative sectional curvature remain negative under a sufficient small perturbation we immediately deduce our main result:

Corollary 1.4. Let $g_{0}$ be an $O(n)$-invariant, asymptotically hyperbolic metric on $\mathbb{R}^{n \geq 9}$ with negative sectional curvatures. There exist continuous multiparameter families $g_{\alpha}$ of non-isometric, mutually isoscattering metrics on $\mathbb{R}^{n}$ which coincide with $g_{0}$ outside a given $O(n)$-invariant compact set and have negative sectional curvatures. 
Guillemin and Kazhdan [16] showed that negatively curved closed surfaces are infinitesimally spectrally rigid. Min-Oo [25] extended this result to $n$-dimensional compact manifolds with negative curvature operator; Croke and Sharafutdinov [7] extended it to $n$-dimensional compact manifolds of strictly negative sectional curvature. All of these results rely on the following basic idea: If $\left\{g_{t}\right\}$ is a one-parameter family of negatively curved metrics with the same Laplace spectrum, then the lengths of closed geodesics are also identical by the Duistermaat-Guillemin trace formula [8]. Thus, the integrals of $\frac{d}{d t} g_{t}$ along any closed geodesic are zero. This condition is then used to prove that $\left\{g_{t}\right\}$ is the orbit of a fixed metric under the action of a one-parameter group of diffeomorphisms.

A crucial ingredient of their proofs is that closed manifolds with strictly negative sectional curvatures have a dense orbit of the geodesic flow in the unit tangent bundle. This argument fails in the case of noncompact manifolds.

Gordon and Szabo [19] already constructed examples of compact manifolds with boundary that have strictly negative sectional curvatures but admit nontrivial isospectral deformations.

The proof of Theorem 1.1 uses the Riemannian submersion method (see, e.g., [10], [9], [13], [30], [31], [32]). The present paper generalizes previous results of Gordon and the first author [11], who constructed continuous families of compactly supported perturbations of the Euclidean metric on $\mathbb{R}^{n}$ with the same scattering phase. Although discrete sets of metrics with the same scattering data have been previously constructed by a number of authors (see [1], [2], [3], [4], [19], [35], [36]), the results of [11] and the present paper are the first examples of continuous families of non-isometric metrics with the same scattering data. Moreover, the metrics in [11] and the present paper are not even locally isometric.

We conclude with a brief sketch of the contents. In $\S 2$, we recall some basic facts regarding the spectral shift function and resolvent resonances. In $\S 3$, we give a version of the Riemannian submersion method adapted to scattering theory. In $\S 4$ we show how to construct examples - among others, those which yield the proof of Theorem 1.1; see remarks 4.8(ii) and 4.9, and in $\S 5$ we show that these examples are nontrivial (i.e., the metrics in the continuous isophasal/isopolar families which we construct are mutually non-isometric).

\section{SCATtering Theory}

2.1. The Spectral Shift. Let $\left(X, g_{0}\right)$ be a complete Riemannian manifold, and let $g$ be another complete metric on $X$. For simplicity we will suppose that the volume forms of $g$ and $g_{0}$ are equal so that the respective Laplacians $\Delta$ and $\Delta_{0}$ act on the same Hilbert spaces. We wish to define the spectral shift function associated to the pair $\left(\Delta, \Delta_{0}\right)$.

The following theorem is a standard consequence of the Birman-Krein theory of the spectral shift (see, for example, [34], chapter 8 for discussion and references, and see particularly Theorem 1 of $\S 8.9 .2$ ).

Theorem 2.1. Suppose that $H$ and $H_{0}$ are positive self-adjoint operators on a Hilbert space, and that $(H+c)^{-k}-\left(H_{0}+c\right)^{-k}$ is a trace-class operator for some $c>0$ and some positive integer $k$. Then there is a real-valued measurable and locally integrable function $\lambda \mapsto \xi\left(\lambda, H, H_{0}\right)$ with the following properties:

(i) $\xi\left(\lambda, H, H_{0}\right)=0$ if $\lambda<0$, 
(ii) For any real-valued function $f \in C^{2}(\mathbb{R})$ that obeys the estimate

$$
\sup _{\lambda \geq 1}\left|\lambda^{1+\varepsilon} \cdot \frac{d}{d \lambda}\left(\lambda^{k+1} f^{\prime}(\lambda)\right)\right|<\infty
$$

for some $\varepsilon>0$, the formula

$$
\operatorname{Tr}\left[f(H)-f\left(H_{0}\right)\right]=-\int \xi\left(\lambda, H, H_{0}\right) f^{\prime}(\lambda) d \lambda
$$

holds.

Remark 2.2. (i) The function $\xi\left(\cdot, H, H_{0}\right)$ is called the spectral shift function associated with $H$ and $H_{0}$.

(ii) Note that the class of admissible functions $f$ includes the function $f(\lambda)=$ $\exp (-t \lambda)$. With this choice of $f$ we see that the formal Laplace transform of the "measure" $d \xi$ is $\operatorname{Tr}\left(\exp (-t H)-\exp \left(-t H_{0}\right)\right)$, a relative heat trace.

Remark 2.3. The spectral shift function is a natural analogue of the counting function for eigenvalues of the Laplacian on a compact manifold. If $(X, g)$ is a compact Riemannian manifold, $H$ is the Laplacian on $(X, g),\left\{\lambda_{k}\right\}_{k=0}^{\infty}$ are the eigenvalues of $H$, and

the 'trace formula'

$$
N(\lambda)=\#\left\{\lambda_{k}: \lambda_{k} \leq \lambda\right\}
$$

$$
\operatorname{Tr}(f(H))=-\int f^{\prime}(\lambda) N(\lambda) d \lambda
$$

holds. In the non-compact case it is necessary to 'renormalize' by the Laplacian of a reference metric in order to obtain a well-defined trace formula.

The following result is easily deduced from [5] and guarantees that the trace condition in Theorem 2.1 is satisfied by the pair $\left(H, H_{0}\right)=\left(\Delta, \Delta_{0}\right)$ under certain assumptions which will always be satisfied in our later constructions (see sections 3 and 4$)$.

Theorem 2.4. Let $X$ be a smooth manifold of dimension $n$ and let $K$ be a compact subset of $X$. Suppose that $g_{0}$ and $g$ are complete metrics on $X$ with the property that $g=g_{0}$ on $X \backslash K$ and the volume forms of $g$ and $g_{0}$ coincide. Let $\Delta$ be the Laplacian on $(X, g)$ and let $\Delta_{0}$ be the Laplacian on $\left(X, g_{0}\right)$. Then, for any $c>0$ and $k>n / 2$, the operator

$$
(\Delta+c)^{-k}-\left(\Delta_{0}+c\right)^{-k}
$$

is a trace-class operator. In particular, there is a spectral shift function $\xi\left(\cdot, \Delta, \Delta_{0}\right)$.

Definition 2.5. Let $g_{0}, g_{1}, g_{2}$ be complete metrics on a manifold $X$ such that both $\left(\Delta_{1}, \Delta_{0}\right)$ and $\left(\Delta_{2}, \Delta_{0}\right)$ satisfy the conditions of Theorem 2.1. We call $g_{1}$ and $g_{2}$ isophasal (with respect to $g_{0}$ ) if the associated spectral shift functions coincide almost everywhere.

The spectral shift function is connected to the scattering phase by the celebrated Birman-Krein formula (see, for example [34], §8.4). Although we will not use the Birman-Krein formula explicitly, we recall some basic ideas to motivate the terminology of Definition 2.5. For a pair of operators $\left(H, H_{0}\right)$ on a Hilbert space $\mathcal{H}$ satisfying the hypotheses of Theorem 2.1, let

$$
W^{ \pm}=s-\lim _{t \rightarrow \pm \infty} \exp (i t H) \exp \left(-i t H_{0}\right) P_{\text {a.c. }}\left(H_{0}\right)
$$


where $s$-lim means limit in the strong operator topology on $\mathcal{H}$, and $P_{\text {a.c. }}\left(H_{0}\right)$ projects onto the absolutely continuous spectral subspace for the operator $H_{0}$. The trace-class theory of scattering asserts that these limits exist and that $\operatorname{Ran} W^{+}=$ $\operatorname{Ran} W^{-}=\mathcal{H}_{\text {a.c. }}(H)$, the absolutely continuous spectral subspace for the operator $H$. Thus the scattering operator $S=\left(W^{+}\right)^{*} W^{-}$is well-defined; owing to the intertwining relation $\exp (i s H) W^{ \pm}=W^{ \pm} \exp \left(i s H_{0}\right)$, the operator $S$ commutes with $H_{0}$. If $\mathcal{H}=\int{ }^{\oplus} \mathfrak{h}_{\lambda} d \lambda$ is a direct integral decomposition of $\mathcal{H}$ into spectral subspaces for $H_{0}$, then $S=\int{ }^{\oplus} S(\lambda) d \lambda$ where $S(\lambda)$ is a unitary operator of determinant class on $\mathfrak{h}_{\lambda}$ (that is, $S(\lambda)-I$ is a trace-class operator so that the operator determinant $\operatorname{det} S(\lambda)$ is well-defined). The Birman-Krein formula asserts that

$$
\operatorname{det} S(\lambda)=\exp \left(2 \pi i \xi\left(\lambda, H, H_{0}\right)\right)
$$

for almost every $\lambda$. The quantity $\arg \operatorname{det} S(\lambda)$ is referred to as the scattering phase, so it is clear that equality of spectral shifts implies equality of scattering phases.

2.2. Resolvent Resonances. Resolvent resonances are defined for many classes of non-compact Riemannian manifolds, and have been extensively studied for one particular class, namely the asymptotically hyperbolic manifolds (see, for example, [20], [22], [23] for spectral theory and meromorphic continuation of Green's function and [17], [18] for bounds on the distribution of resonances). Let $(X, g)$ be a conformally compact Riemannian manifold, that is, $X$ is the interior of a smooth manifold $\bar{X}$ with boundary, and there is a defining function $\rho$ for $\partial \bar{X}$ so that $h=\rho^{2} g$ extends to a smooth, nondegenerate metric on $\bar{X}$ (the condition that $\rho$ be a defining function for $\partial \bar{X}$ means that $\rho \in \mathcal{C}^{\infty}(\bar{X}), \rho \geq 0, \rho^{-1}(0)=\partial \bar{X}$, and for each $x \in \partial \bar{X}, d \rho(x) \neq 0)$. Such a manifold $(X, g)$ is called asymptotically hyperbolic if, in addition, $|d \rho(x)|_{h} \rightarrow 1$ as $x \rightarrow \partial \bar{X}$. An elementary example is the open unit ball in $\mathbb{R}^{n}$ with the metric $g=\rho^{-2} h$, where $h$ is the Euclidean metric restricted to the open unit ball in $\mathbb{R}^{n}$ and $\rho(x)=\frac{1}{2}\left(1-|x|^{2}\right)$.

If $(X, g)$ is asymptotically hyperbolic and $\operatorname{dim} X=n$, then the Laplacian $\Delta_{g}$ has at most finitely many $L^{2}$-eigenvalues of finite multiplicity in the interval $[0,(n-$ $1)^{2} / 4$ ) [22] and absolutely continuous spectrum in the interval $\left[(n-1)^{2} / 4, \infty\right.$ ) (this follows from the meromorphic continuation of the resolvent kernel established in [23]). It follows that the resolvent operator

$$
\widetilde{R}(z)=\left(\Delta_{g}-z\right)^{-1}
$$

is a meromorphic function of $z$ on the cut plane $\mathbb{C} \backslash\left[(n-1)^{2} / 4, \infty\right)$ with at most finitely many poles of finite rank at the eigenvalues (if any) of $\Delta_{g}$. Viewed as an operator from $L^{2}(X, g)$ to itself, the resolvent $\widetilde{R}(z)$ becomes unbounded as $\operatorname{Im}(z) \rightarrow$ 0 and $\operatorname{Re}(z)$ approaches any number $\lambda \in\left[(n-1)^{2} / 4, \infty\right)$ owing to the presence of the absolutely continuous spectrum. On the other hand, the operator $R(z)$ has a Schwarz kernel (Green's function) with respect to the Riemannian measure on $(X, g)$ which continues meromorphically in $z$ to a double cover of the cut plane. This meromorphic continuation can be captured by viewing $\widetilde{R}(z)$ as a map from a smaller space than $L^{2}(X, g)$, namely the $L^{2}$ functions of compact support, to a larger space, the locally square integrable functions on $X$. Poles of the meromorphically continued Green's function are called resolvent resonances and physically represent "almost bound states." As with eigenvalues of the Laplacian, resolvent resonances determine the expansion in "normal modes" of solutions for the wave equation but unlike eigenvalues they represent "normal modes" whose energy in any bounded 
region decays with time (see, for example, [6], [33], and [37] for recent work on such "resonance wave expansions"). The energy decay is a consequence of the non-compactness of the underlying manifold.

To make these notions more precise, let

$$
R(s)=(\Delta-s(n-1-s))^{-1}
$$

for $\operatorname{Re}(s)>(n-1) / 2$. The map $s \mapsto s(n-1-s)$ maps this region onto the cut plane $\mathbb{C} \backslash\left[(n-1)^{2} / 4, \infty\right)$. In the half-plane $\operatorname{Re}(s)>(n-1) / 2, R(s)$ is a meromorphic operator-valued function with poles at those real numbers $\zeta>(n-1) / 2$ for which $\zeta(n-1-\zeta)$ is an eigenvalue of $\Delta$. Denote by $L_{\text {comp }}^{2}(X, g)$ the compactly supported, square-integrable functions on $(X, g)$ and by $L_{\text {loc }}^{2}(X, g)$ the space of measurable, locally square-integrable functions on $(X, g)$. Mazzeo and Melrose [23] proved:

Theorem 2.6. Suppose that $(X, g)$ is an asymptotically hyperbolic manifold of dimension $n$. Then $R(s)$, viewed as an operator from $L_{\text {comp }}^{2}(X, g)$ to $L_{\text {loc }}^{2}(X, g)$, has a meromorphic continuation to the complex s-plane whose poles do not accumulate in the complex s-plane with the possible exception of the points

$$
\{(n-1-j) / 2: j=1,2, \cdots\} \text {. }
$$

The operator $R(s)$ has no poles on the line $\operatorname{Re}(s)=(n-1) / 2$ with the possible exception of the point $s=(n-1) / 2$, where the Laurent expansion has finite polar part. If $\zeta$ is a pole of $R(s)$ and $\zeta$ is not an exceptional point as above, then the resolvent $R(s)$ has a Laurent series with finite polar part of the form

$$
\sum_{j=1}^{N_{\zeta}} A_{j}(s-\zeta)^{-j}
$$

where the $A_{j}$ are finite-rank operators from $L_{\text {comp }}^{2}(X, g)$ to $L_{\mathrm{loc}}^{2}(X, g)$.

Remark 2.7. Guillopé-Zworski [18] showed that there are no exceptional points if $(X, g)$ has constant sectional curvatures outside a compact subset of $X$, and Guillarmou [14] has given necessary and sufficient conditions for the meromorphically continued resolvent operator $R(s)$ to have essential singularities at the exceptional points. For a careful analysis of the relationship between resolvent poles and poles of the meromorphically continued scattering operator, see [15].

Note that, for poles $\zeta$ of $R(s)$ with $s>(n-1) / 2, R(s)$ has polar part

$$
A_{1}(n-1-2 \zeta)^{-1}(s-\zeta)^{-1}
$$

where $A_{1}$ is the orthogonal projection onto the eigenspace of $\Delta$ with eigenvalue $\zeta(n-1-\zeta)$ (the factor $(n-1-2 \zeta)$ is the derivative of the map $s \mapsto s(n-1-s)$ at $s=\zeta)$. Thus, $\operatorname{dim}\left(\operatorname{Ran} A_{1}\right)$ is the multiplicity of the eigenvalue associated to $\zeta$. More generally, the multiplicity of a resolvent pole is the number

$$
m_{\zeta}=\operatorname{dim}\left(\operatorname{Ran} P_{\zeta}\right)
$$

where

$$
P_{\zeta}=\int_{|s-\zeta|<\varepsilon}(2 s-n+1) R(s) d s
$$

and $\varepsilon>0$ is chosen so small that there are no other resolvent resonances in the disc of size $\varepsilon$ centered at $\zeta$. Note that, if $\zeta$ is a pole of the resolvent corresponding 
to an $L^{2}$-eigenvalue of the Laplacian, $m_{\zeta}$ is the dimension of the corresponding eigenspace.

Definition 2.8. Two asymptotically hyperbolic manifolds are called isopolar if the associated Laplacians on functions have the same eigenvalues and resolvent poles with multiplicity.

\section{Isoscattering via Effective Torus Actions}

In this section, we prove two theorems (Theorems 3.2 and 3.3) which adapt Theorem 1.4 of [32] to the setting of scattering theory on a complete, non-compact Riemannian manifold. The first gives a criterion for constructing isophasal manifolds and the second gives a criterion for constructing isopolar manifolds.

To adapt the proof of Theorem 1.4 of [32] to the scattering context, it will be helpful to recall how the Laplacian is defined via quadratic forms on a closed manifold, a manifold with boundary, and an open manifold. In all cases we begin with the positive quadratic form

$$
\mathfrak{q}(u, v)=\int_{X} \nabla u \cdot \nabla v \mathrm{dvol}_{g}
$$

for $u$ and $v$ belonging to a suitably chosen linear space $\mathfrak{Q}$ of smooth functions, and we introduce the auxiliary inner product

$$
\langle u, v\rangle=\mathfrak{q}(u, v)+(u, v)_{L^{2}(X, g)}
$$

on $\mathfrak{Q}$. The Laplacian is then defined as the Friedrichs extension associated to the quadratic form $\mathfrak{q}$ (see, for example, Reed-Simon [28], §X.3, Theorem X.23; the operator $A$ referred to there is in our case the Laplace operator on $\mathfrak{Q}$ ). The Friedrichs extension has the property that its operator domain $\mathfrak{D}$ is contained in the form domain of the closure of $\mathfrak{q}$. By the Form Representation Theorem (see for example Kato [21], Theorem VI.2.1), a vector $\varphi$ belongs to $\mathfrak{D}$ if and only if there is a vector $\chi \in L^{2}(X, g)$ with the property that

$$
\mathfrak{q}(\varphi, \eta)=(\chi, \eta)
$$

for all $\eta \in \mathfrak{Q}$, where the right-hand side denotes the $L^{2}$-inner product. In this case $\Delta \varphi=\chi$.

(1) If $X$ is a closed manifold, the closure of the form $\mathfrak{q}$ with domain $\mathfrak{Q}=\mathcal{C}^{\infty}(X)$ has form domain $H^{1}(X, g)$, a Hilbert space with inner product given by $(3.2)$, and the self-adjoint operator associated to $\mathfrak{q}$ is the usual Laplacian on functions.

(2) If $X$ is a compact manifold with boundary, as in [32], the quadratic form (3.1) with domain $\mathfrak{Q}=\mathcal{C}_{0}^{\infty}(X)$ closes to the quadratic form associated with the Laplacian on $X$ with Dirichlet boundary conditions, while the form (3.1) with domain $\mathfrak{Q}=\mathcal{C}^{\infty}(X)$ closes to the quadratic form of the Laplacian with Neumann boundary conditions on $X$. The domain of the closure of $\mathfrak{q}$ is the space $H_{0}^{1}(X, g)$ in the case of Dirichlet boundary conditions, and $H^{1}(X, g)$ in the case of Neumann boundary conditions.

(3) If $X$ is an open manifold and $\left(X, g_{0}\right)$ is complete, the quadratic form $\mathfrak{q}$ with domain $\mathfrak{Q}=\mathcal{C}_{0}^{\infty}(X)$ is closable and the domain of its closure is again denoted $H^{1}(X, g)$. The Laplacian on $X$ is the unique positive operator associated to the closure of $\mathfrak{q}$ by the Friedrichs construction. 
In [32], manifolds $(X, g)$ and $\left(X^{\prime}, g^{\prime}\right)$ (which may be closed manifolds or compact manifolds with boundary) are shown to be isospectral by constructing an isometry $Q$ from $H_{*}^{1}(X, g)$ to $H_{*}^{1}\left(X^{\prime}, g^{\prime}\right)$ which extends to a unitary map from $L^{2}(X, g)$ to $L^{2}\left(X^{\prime}, g^{\prime}\right)$ (here $H_{*}^{1}(X, g)$ denotes one of $H_{0}^{1}(X, g)$ or $H^{1}(X, g)$ and the same choice is made for $\left.H_{*}^{1}\left(X^{\prime}, g^{\prime}\right)\right)$. Here we observe that the same construction works if $(X, g)$ and $\left(X^{\prime}, g^{\prime}\right)$ are open manifolds, with some additional hypotheses to insure that scattering data are preserved.

We first recall Theorem 1.4 of [32] (with trivial notational changes). By a torus we mean a nontrivial, compact, connected abelian Lie group. Suppose that $T$ is a torus acting smoothly and effectively by isometries on a complete Riemannian manifold $(X, g)$, and let $\widehat{X}$ denote the union of principal orbits of $T$. Then $\widehat{X}$ is an open and dense submanifold of $X$. We denote by $g^{T}$ the unique Riemannian metric on $\widehat{X} / T$ such that the canonical projection $\pi:(\widehat{X}, g) \rightarrow\left(\widehat{X} / T, g^{T}\right)$ is a Riemannian submersion.

Theorem 3.1. [32] Let $T$ be a torus which acts effectively on two compact connected Riemannian manifolds $\left(X_{1}, g_{1}\right)$ and $\left(X_{2}, g_{2}\right)$ by isometries. For each subtorus $W \subset$ $T$ of codimension one, suppose there exists a T-equivariant diffeomorphism $F_{W}$ : $X_{1} \rightarrow X_{2}$ which satisfies:

(i) $F_{W}^{*} \mathrm{dvol}_{2}=\mathrm{dvol}_{1}$ and

(ii) $F_{W}$ induces an isometry $\bar{F}_{W}$ between the quotient manifolds $\left(\widehat{X}_{1} / W, g_{1}^{W}\right)$ and $\left(\widehat{X}_{2} / W, g_{2}^{W}\right)$.

Then $\left(X_{1}, g_{1}\right)$ and $\left(X_{2}, g_{2}\right)$ are isospectral; if the manifolds have boundary then they are Dirichlet and Neumann isospectral.

To construct examples of manifolds with the same scattering phase, we consider a noncompact complete Riemannian manifold $\left(X, g_{0}\right)$ and metrics $g_{1}$ and $g_{2}$ (on the same smooth manifold $X$ ) which are compactly supported perturbations of $g_{0}$ For simplicity, we suppose that $g_{1}$ and $g_{2}$ coincide with $g_{0}$ outside a fixed compact subset of $X$. The metric $g_{0}$ serves as a "background" or "comparison" metric. The following Theorem gives conditions under which the scattering phases of two pairs $\left(\Delta_{1}, \Delta_{0}\right)$ and $\left(\Delta_{2}, \Delta_{0}\right)$ are the same. In order to guarantee that the torus action is compatible with the background metric $g_{0}$, we add an hypothesis about the map $F_{W}$.

Theorem 3.2. Let $X$ be a smooth Riemannian manifold carrying complete metrics $g_{0}, g_{1}$, and $g_{2}$, where the $g_{i}$ are compactly supported perturbations of $g_{0}$. Suppose that the volume forms of $g_{0}, g_{1}, g_{2}$ are equal, so that the Laplacians $\Delta_{0}, \Delta_{1}$, and $\Delta_{2}$ act on the same Hilbert space. Let $T$ be a torus which acts effectively by isometries on $\left(X, g_{1}\right)$ and $\left(X, g_{2}\right)$. For each subtorus $W \subset T$ of codimension one, suppose that there exists a $T$-equivariant diffeomorphism $F_{W}$ of $X$ with the following properties:

(i) $F_{W}^{*}\left(\operatorname{dvol}_{0}\right)=\mathrm{dvol}_{0}$,

(ii) $F_{W}$ induces an isometry $\bar{F}_{W}$ between the quotient manifolds $\left(\widehat{X} / W, g_{1}^{W}\right)$ and $\left(\widehat{X} / W, g_{2}^{W}\right)$, and

(iii) $F_{W}^{*}: \mathcal{C}^{\infty}(X) \rightarrow \mathcal{C}^{\infty}(X)$ commutes with $\Delta_{0}$.

Then $g_{1}$ and $g_{2}$ are isophasal with respect to $g_{0}$ (see Definition 2.5).

Proof. The space $\mathcal{H}=L^{2}\left(X, g_{0}\right)$ admits a decomposition

$$
\mathcal{H}=\mathcal{H}_{0} \oplus \bigoplus_{W \subset T}\left(\mathcal{H}_{W} \ominus \mathcal{H}_{0}\right)
$$


where $\mathcal{H}_{0}$ denotes the $T$-invariant functions, $\mathcal{H}_{W}$ denotes the $W$-invariant functions, and the direct sum goes over subtori $W$ of $T$ having codimension one. Denote one of the various maps $F_{W}$ by $F_{0}$. The proof of Theorem 1.4 in [32] goes over without change to show that there is an isometry $Q$ from $\mathcal{H}$ to $\mathcal{H}$ given by

$$
Q=F_{0}^{*-1} \oplus \bigoplus_{W \subset T} F_{W}^{*-1}
$$

which restricts to a unitary map from $H^{1}\left(X, g_{1}\right)$ to $H^{1}\left(X, g_{2}\right)$.

We claim that (a) $Q$ commutes with $\left(\Delta_{0}-z\right)^{-1}$ and (b) the identity

$$
Q\left(\Delta_{1}-z\right)^{-1}=\left(\Delta_{2}-z\right)^{-1} Q
$$

holds for any $z \in \mathbb{C} \backslash \mathbb{R}$. Claim (a) follows directly from hypothesis (iii) and the definition of $Q$. To prove claim (b), we note that, since $Q$ is an isometry from $H^{1}\left(X, g_{1}\right)$ to $H^{1}\left(X, g_{2}\right)$, we have

$$
\mathfrak{q}_{2}(Q \varphi, \psi)=\mathfrak{q}_{1}\left(\varphi, Q^{-1} \psi\right)
$$

for any $\varphi$ in the form domain of $\Delta_{1}$ and $\psi$ belonging to the form domain of $\Delta_{2}$. We will use this relation and the form representation theorem (see (3.3) and the surrounding remarks) to prove that if $\varphi$ belongs to $\mathfrak{D}\left(\Delta_{1}\right)$ then $Q \varphi \in \mathfrak{D}\left(\Delta_{2}\right)$ and $\Delta_{2}(Q \varphi)=Q\left(\Delta_{1} \varphi\right)$. Since $\varphi \in \mathfrak{D}\left(\Delta_{1}\right)$, there is a vector $\chi \in L^{2}\left(X, g_{1}\right)$ with the property that

$$
\mathfrak{q}_{1}(\varphi, \eta)=(\chi, \eta)_{L^{2}\left(X, g_{1}\right)}
$$

for any $\eta$ belonging to the form domain of $\Delta_{1}$; by definition, $\Delta_{1} \varphi=\chi$. It follows from (3.5) that for any $\zeta$ in the form domain of $\Delta_{2}$,

$$
\begin{aligned}
\mathfrak{q}_{2}(Q \varphi, \zeta) & =\mathfrak{q}_{1}\left(\varphi, Q^{-1} \zeta\right) \\
& =\left(\chi, Q^{-1} \zeta\right)_{L^{2}\left(X, g_{1}\right)} \\
& =(Q \chi, \zeta)_{L^{2}\left(X, g_{2}\right)}
\end{aligned}
$$

so that $Q \varphi \in \mathfrak{D}\left(\Delta_{2}\right)$ and $\Delta_{2}(Q \varphi)=Q \chi$ as claimed. We now conclude that $Q\left(\Delta_{1}-z\right)^{-1}=\left(\Delta_{2}-z\right)^{-1} Q$, proving claim (b). From claims (a) and (b) we deduce by differentiation in $z$ that

$$
Q\left(\Delta_{0}-z\right)^{-m}=\left(\Delta_{0}-z\right)^{-m} Q
$$

and

$$
Q\left(\Delta_{1}-z\right)^{-m}=\left(\Delta_{2}-z\right)^{-m} Q
$$

for any integer $m$ and any $z \in \mathbb{C} \backslash \mathbb{R}$. By the Stone-Weierstrass Theorem, polynomials in $(x \pm i)^{-1}$ are dense in the Banach algebra $\mathcal{C}_{\infty}(\mathbb{R})$ of continuous functions vanishing at infinity. It now follows that for any $f \in \mathcal{C}_{\infty}(\mathbb{R}), \quad Q f\left(\Delta_{1}\right)=f\left(\Delta_{2}\right) Q$ and $Q f\left(\Delta_{0}\right)=f\left(\Delta_{0}\right) Q$.

For $t>0$, let $f_{t} \in \mathcal{C}_{\infty}(\mathbb{R})$ with $f_{t}(x)=\exp (-t x)$ for $x \geq 0$. Since

$$
\begin{aligned}
\operatorname{Tr}\left(f_{t}\left(\Delta_{1}\right)-f_{t}\left(\Delta_{0}\right)\right) & =\operatorname{Tr}\left(Q\left[f_{t}\left(\Delta_{1}\right)-f_{t}\left(\Delta_{0}\right)\right] Q^{*}\right) \\
& =\operatorname{Tr}\left(f_{t}\left(\Delta_{2}\right)-f_{t}\left(\Delta_{0}\right)\right),
\end{aligned}
$$

we conclude from Theorems 2.1 and 2.4 , Remark 2.2, and the Krein trace formula (2.1) that for any $t>0$,

$$
\int \xi\left(\lambda, \Delta_{1}, \Delta_{0}\right) \exp (-\lambda t) d \lambda=\int \xi\left(\lambda, \Delta_{2}, \Delta_{0}\right) \exp (-\lambda t) d \lambda .
$$

We again appeal to the Stone-Weierstrass theorem to argue that polynomials in $\exp (-\lambda t)$ for $t>0$ are dense in the continuous functions on $\mathbb{R}^{+}$that vanish at 
infinity, so that for any continuous function $f$ of compact support in $(0, \infty)$ we have

$$
\int \xi\left(\lambda, \Delta_{1}, \Delta_{0}\right) f(\lambda) d \lambda=\int \xi\left(\lambda, \Delta_{2}, \Delta_{0}\right) f(\lambda) d \lambda .
$$

It now follows that $\xi\left(\lambda, \Delta_{1}, \Delta_{0}\right)=\xi\left(\lambda, \Delta_{2}, \Delta_{0}\right)$ almost everywhere.

The next theorem concerns resolvent resonances. To discuss these, we impose an additional assumption on the map $F_{W}$ which allows us to construct an intertwining operator $Q$ for the meromorphically continued resolvents.

Theorem 3.3. $L e t g_{1}$ and $g_{2}$ be asymptotically hyperbolic metrics on a manifold $X$. Suppose that the volume forms of $g_{1}, g_{2}$ are equal, so that the Laplacians $\Delta_{1}$ and $\Delta_{2}$ act on the same Hilbert space. Let $T$ be a torus which acts effectively by isometries on $\left(X, g_{1}\right)$ and $\left(X, g_{2}\right)$. For each subtorus $W \subset T$ of codimension one, suppose that there exists a T-equivariant diffeomorphism $F_{W}$ of $X$ with the following properties:

(i) $F_{W}^{*}(\mathrm{dvol})=\mathrm{dvol}$, where dvol $:=\mathrm{dvol}_{1}=\mathrm{dvol}_{2}$.

(ii) $F_{W}$ induces an isometry $\bar{F}_{W}$ between the quotient manifolds $\left(\widehat{X} / W, g_{1}^{W}\right)$ and $\left(\widehat{X} / W, g_{2}^{W}\right)$, and

(iii) If $C$ is a compact subset of $X$, then $\cup_{W \subset T} F_{W}(C)$ and $\cup_{W \subset T} F_{W}^{-1}(C)$ are also compact, where the union goes over subtori of $T$ having codimension one.

Then $\left(X, g_{1}\right)$ and $\left(X, g_{2}\right)$ are isopolar (see Definition 2.8).

Proof. First, we argue as in the proof of Theorem 3.2 that, under assumptions (i) and (ii), there is a unitary operator $Q$ which intertwines the resolvents of $\Delta_{1}$ and $\Delta_{2}$. Next, we show that the operator $Q$ preserves the space $L_{\text {comp }}^{2}(X)$ (since the volume forms of $g_{1}$ and $g_{2}$ are equal, we denote either of the spaces $L_{\text {comp }}^{2}\left(X, g_{1}\right)$ or $L_{\text {comp }}^{2}\left(X, g_{2}\right)$ by $\left.L_{\text {comp }}^{2}(X)\right)$. To see this, suppose that $f$ has support contained in a fixed subset $C$ of $X$, which we will assume without loss is $T$-invariant. The projection of $f$ onto the subspace $\mathcal{H}_{0}$ of $T$-invariant functions is the average $\int_{T} f(z \cdot x) d \mu_{T}(z)$, where $d \mu_{T}$ is normalized Haar measure on $T$, and so is clearly supported in $C$. A similar formula computes the projection of $f$ onto the subspace $\mathcal{H}_{W}$ of $W$-invariant functions for any subtorus $W$ of $T$. It now follows from assumption (iii) that $Q f$ and $Q^{-1} f$ have support in a fixed compact subset of $X$. Hence, $Q$ and $Q^{-1}$ preserve the functions of compact support on $X$ and, since $Q$ is a unitary mapping from $L^{2}\left(X, g_{1}\right)$ to $L^{2}\left(X, g_{2}\right)$, they also preserve the space $L_{\text {comp }}^{2}(X)$. For $j=1,2$, let

$$
R_{j}(s)=\left(\Delta_{j}-s(n-1-s)\right)^{-1}
$$

For all $u, v \in L^{2}(X)$ and all $s$ with $\operatorname{Re}(s)>(n-1) / 2$ we have $\left(Q u, Q R_{1}(s) v\right)=$ $\left(Q u, R_{2}(s) Q v\right)$ and thus

$$
\left(u, R_{1}(s) v\right)=\left(Q u, R_{2}(s) Q v\right) .
$$

Now we assume that $u$ and $v$ - and hence, also, $Q u$ and $Q v$ - belong to $L_{\text {comp }}^{2}(X)$. Then, by Theorem 2.6, the functions on each side of (3.6) admit a meromorphic continuation to the complex $s$-plane. If $\zeta$ is not an exceptional point, the resolvents $R_{1}(s)$ and $R_{2}(s)$ have finite polar parts at $s=\zeta$ whose coefficients are finite-rank operators from $L_{\text {comp }}^{2}(X)$ to $L_{\text {loc }}^{2}(X)$ (see $(2.2)$ ). For $j=1,2$, let

$$
P_{\zeta, j}=\int_{|s-\zeta|<\varepsilon}(2 s-n+1) R_{j}(s) d s
$$


where $\varepsilon>0$ is so small that $\zeta$ is the only resonance in the disc of radius $\varepsilon$ about $\zeta$. Recall that the multiplicity of the resonance $\zeta$ for $\Delta_{j}$ is given by $m_{\zeta, j}=$ $\operatorname{dim}\left(\operatorname{Ran} P_{\zeta, j}\right)$. From (3.6) we conclude that

$$
\left(u, P_{\zeta, 1} v\right)=\left(Q u, P_{\zeta, 2} Q v\right)
$$

for all $u, v \in L_{\text {comp }}^{2}(X)$. Let $v_{1}, \ldots, v_{m_{\zeta, 1}} \in L_{\text {comp }}^{2}(X)$ be such that the corresponding functions $w_{j}:=P_{\zeta, 1} v_{j} \in L_{\text {loc }}^{2}(X)$ constitute a basis of $\operatorname{Ran} P_{\zeta, 1}$. By an elementary argument, there exist $u_{1}, \ldots, u_{m_{\zeta, 1}} \in L_{\text {comp }}^{2}(X)$ such that the matrix $\left(\left(u_{i}, w_{j}\right)\right)$ is invertible. By (3.7) it follows that $\left(\left(Q u_{i}, P_{\zeta, 2} Q v_{j}\right)\right)$ is invertible. Thus the functions $P_{\zeta, 2} Q v_{j}$ are linearly independent in $L_{\text {loc }}^{2}(X)$. We conclude $m_{\zeta, 1} \leq m_{\zeta, 2}$. Replacing $u, v$ by $Q^{-1} u, Q^{-1} v$ in (3.7), we analogously obtain $m_{\zeta, 2} \leq m_{\zeta, 1}$.

\section{Applications}

In Theorems 4.3, 4.7 and Remarks 4.8, 4.10 we will formulate a succesive series of specializations of Theorems 3.2 and 3.3, leading up to the examples which give us the proof of Theorem 1.1.

Let $\left(X, g_{0}\right)$ be a complete Riemannian manifold, and let $T \subset \operatorname{Isom}\left(X, g_{0}\right)$ be a torus with Lie algebra $\mathfrak{t}$. For $Z \in \mathfrak{t}$, we denote the induced vectorfield on $X$ by $Z^{*}$.

Definition 4.1. $\quad$ (i) $A \mathfrak{t}$-valued 1-form $\lambda$ on $X$ is called admissible (with respect to the $T$-action) if $\lambda$ is T-invariant and horizontal, i.e., vanishes on vectors tangent to the T-orbits.

(ii) With each admissible $\lambda$ on $X$ we associate a Riemannian metric $g_{\lambda}$ on $X$ defined by

$$
g_{\lambda}(X, Y)=g_{0}\left(X+\lambda(X)^{*}, Y+\lambda(Y)^{*}\right) .
$$

(iii) Two admissible $\mathfrak{t}$-valued 1 -forms $\lambda, \lambda^{\prime}$ on $X$ are called isospectral (with respect to $g_{0}$ and the $T$-action) if for each $\mu \in \mathfrak{t}^{*}$ there exists a T-equivariant isometry $F_{\mu} \in \operatorname{Isom}\left(X, g_{0}\right)$ such that $\mu \circ \lambda=F_{\mu}^{*}\left(\mu \circ \lambda^{\prime}\right)$.

Remark 4.2. $\quad$ (i) If $\lambda, \lambda^{\prime}$ are admissible and isospectral, then so are $\psi \lambda, \psi \lambda^{\prime}$, where $\psi$ is any smooth function on $X$ which is invariant under $T$ and for which the $F_{\mu}$ in the above definition can be chosen such that $\psi$ is invariant under each of them.

(ii) If $\lambda$ is admissible and compactly supported then $g_{\lambda}$ is a compactly supported perturbation of the metric $g_{0}$ on $X$, and the volume elements of $g_{\lambda}$ and $g_{0}$ coincide. Moreover, $g_{0}^{T}=g_{\lambda}^{T}$ on $\widehat{X} / T$.

Theorem 4.3. Let $\lambda, \lambda^{\prime}$ be admissible, isospectral, and compactly supported $\mathfrak{t}$-valued 1 -forms on $\left(X, g_{0}\right)$, where $g_{0}$ is a complete Riemannian metric on $X$. Then $g_{\lambda}$ and $g_{\lambda^{\prime}}$ are isophasal with respect to $g_{0}$. If, moreover, $\left(X, g_{0}\right)$ - and hence also $\left(X, g_{\lambda}\right)$ and $\left(X, g_{\lambda^{\prime}}\right)$ - are asymptotically hyperbolic, then $\left(X, g_{\lambda}\right)$ and $\left(X, g_{\lambda^{\prime}}\right)$ are isopolar.

Proof. We appeal to Theorems 3.2 and 3.3, respectively. Let $W \subset T$ be a subtorus of codimension one. Choose $\mu \in \mathfrak{t}^{*}$ such that $\operatorname{ker} \mu=T_{e} W$, and let $F_{W}:=F_{\mu}$, where $F_{\mu}$ is chosen as in Definition 4.1(iii). Since $F_{\mu}$ is an isometry of $g_{0}$, it commutes with $\Delta_{0}$ and satisfies $F_{\mu}^{*} \operatorname{dvol}_{g_{\lambda^{\prime}}}=\operatorname{dvol}_{g_{\lambda}}$ because of $\operatorname{dvol}_{g_{\lambda}}=\operatorname{dvol}_{g_{0}}=\operatorname{dvol}_{g_{\lambda^{\prime}}}$. We claim that $F_{\mu}$ induces an isometry from $\left(\widehat{X} / W, g_{\lambda}^{W}\right)$ to $\left(\widehat{X} / W, g_{\lambda^{\prime}}^{W}\right)$. Denote the space of vectors in $T_{x} X$ tangent to the $W$-orbit through $x$ by $\mathfrak{w}_{x}$. Let $V \in T_{x} X$ be any vector which is $g_{\lambda}$-orthogonal to $\mathfrak{w}_{x}$. Then $V=V_{0}-\lambda\left(V_{0}\right)$ for some 
$V_{0} \in T_{x} X$ which is $g_{0}$-orthogonal to $\mathfrak{w}_{x}$. We have $F_{\mu *} V=F_{\mu *}\left(V_{0}-\lambda\left(V_{0}\right)^{*}\right)=$ $F_{\mu *} V_{0}-\lambda\left(V_{0}\right)^{*}$, and by the choice of $F_{\mu}$ this is, up to an error in $\mathfrak{w}_{F_{\mu} x}$, the same as $V^{\prime}:=F_{\mu *} V_{0}-\lambda^{\prime}\left(F_{\mu *} V_{0}\right)^{*}$. But $F_{\mu *} V_{0}$ is $g_{0}$-orthogonal to $\mathfrak{w}_{F_{\mu}(x)}$; thus $V^{\prime}$ is the projection of $F_{\mu *} V_{0}$ to the $g_{\lambda^{\prime}}$-orthogonal complement of $\mathfrak{w}_{F_{\mu}(x)}$. Our claim now follows from $\left\|V^{\prime}\right\|_{g_{\lambda^{\prime}}}=\left\|F_{\mu *} V_{0}\right\|_{g_{0}}=\left\|V_{0}\right\|_{g_{0}}=\|V\|_{g_{\lambda}}$.

We have now shown that $F_{\mu}$ satisfies all conditions of Theorem 3.2, as well as conditions (i) and (ii) of Theorem 3.3. It remains to check condition (iii) of the latter. Let $C \subset X$ be compact, and denote by $D$ the maximal $g_{0}$-distance between points of $C$ and points of the compact support $\operatorname{supp}(\lambda)$ of $\lambda$. For any $\mu \in \mathfrak{t}^{*}$ with $\mu \circ \lambda \neq 0$, the set $F_{\mu}(C)$ is then contained in $\left\{x \in X \mid d\left(x, F_{\mu}(\operatorname{supp}(\mu \circ \lambda)) \leq D\right\}=\right.$ $\left\{x \in X \mid d\left(x, \operatorname{supp}\left(\mu \circ \lambda^{\prime}\right)\right) \leq D\right\} \subset\left\{x \in X \mid d\left(x, \operatorname{supp}\left(\lambda^{\prime}\right)\right) \leq D\right\}$. Moreover, for any $\mu$ with $\mu \circ \lambda=0$ we necessarily have $\mu \circ \lambda^{\prime}=0$ and can thus assume $F_{\mu}=$ Id. Hence each $F_{\mu}$ maps $C$ to the union of $C$ and the closed $D$-neighborhood of $\operatorname{supp}\left(\lambda^{\prime}\right)$, which is compact. Thus, the maps $F_{\mu}$ satisfy the required condition; the same argument with reversed roles of $\lambda$ and $\lambda^{\prime}$ works for the maps $F_{\mu}^{-1}$.

In the following, let $H$ be a compact connected semisimple Lie group with Lie algebra $\mathfrak{h}$, and let the Lie algebra $\mathfrak{t}$ of $T$ be endowed with a fixed (auxiliary) Euclidean inner product.

Definition 4.4. $\quad$ (i) Two linear maps $j, j^{\prime}: \mathfrak{t} \rightarrow \mathfrak{h}$ are called isospectral if for each $Z \in \mathfrak{t}$ there is $a_{Z} \in H$ such that $j_{Z}^{\prime}=\operatorname{Ad}_{a_{Z}}\left(j_{Z}\right)$. Here $j_{Z}$ denotes $j(Z)$, and similarly for $j^{\prime}$.

(ii) $j$ and $j^{\prime}$ are called equivalent if there is $\Phi \in \operatorname{Aut}(\mathfrak{h})$ and $C \in \mathrm{O}(\mathfrak{t})$ such that $j_{Z}^{\prime}=\Phi\left(j_{C(Z)}\right)$ for all $Z \in \mathfrak{t}$.

Remark 4.5. Let $\mathfrak{t}=\mathbb{R}^{2}$, equipped with the standard metric, and denote by $\mathcal{J}$ the vector space of all linear maps from $\mathfrak{t}$ to $\mathfrak{h}$.

(i) [13] If $\mathfrak{h}=\mathfrak{s o}(m)$, where $m$ is any positive integer other than $1,2,3,4$, or 6 , then there is a Zariski open subset $\mathcal{O}$ of $\mathcal{J}$ such that each $j \in \mathcal{O}$ belongs to a d-parameter family of isospectral, inequivalent elements of $\mathcal{J}$. Here $d \geq m(m-1) / 2-[m / 2]([m / 2]+2)>1$. For $m=6$, there exist at least 1 -parameter families in $\mathcal{J}$ with these properties.

(ii) [32] If $\mathfrak{h}=\mathfrak{s u}(m)$, where $m \geq 3$, then there is a Zariski open subset $\mathcal{O}$ of $\mathcal{J}$ such that each $j \in \mathcal{O}$ belongs to a continuous family of isospectral, inequivalent elements of $\mathcal{J}$.

(iii) [27] Recently, Emily Proctor established results analogous to those in (i) (multiparameter families) for $\mathfrak{s u}(m \geq 5)$ and $\mathfrak{s p}(m \geq 2)$.

Now suppose that $H \times T$ acts on a complete connected Riemannian manifold $\left(X, g_{0}\right)$ by isometries.

Definition 4.6. For each linear map $j: \mathfrak{t} \rightarrow \mathfrak{h}$ define an associated $\mathfrak{t}$-valued 1 -form $\lambda^{j}$ on $X$ by

$$
\left\langle\lambda^{j}(Y), Z\right\rangle=g_{0}\left(j_{Z}^{*}(x), Y\right)
$$

for all $Z \in \mathfrak{t}$ and $Y \in T_{x} X, x \in X$, where $\langle$,$\rangle is the given inner product on \mathfrak{t}$. Here again, the star denotes the induced vectorfield on $X$.

Theorem 4.7. Let $H \times T$ act on $\left(X, g_{0}\right)$ by isometries. Let $j, j^{\prime}: \mathfrak{t} \rightarrow \mathfrak{h}$ be isospectral, and let $\psi \in \mathcal{C}^{\infty}(X)$ be compactly supported and invariant under $H \times T$. 
(i) If, for each $x \in X$, the $H$-orbit through $x$ and the $T$-orbit through $x$ meet $g_{0}$-orthogonally in $x$, then the $\mathfrak{t}$-valued 1 -forms $\psi \lambda^{j}$ and $\psi \lambda^{j^{\prime}}$ satisfy the conditions of Theorem 4.3.

(ii) Let $\left\{Z_{1}, \ldots, Z_{k}\right\}$ be a basis of $\mathfrak{t}$, and denote by $\lambda_{h}^{j}, \lambda_{h}^{j^{\prime}}$ the horizontal part of $v \lambda^{j}, v \lambda^{j^{\prime}}$, respectively, where $v:=\left\|Z_{1}^{*} \wedge \ldots \wedge Z_{k}^{*}\right\|^{2}$ and the norm on $\wedge^{k} T_{x} M$ is the usual Euclidean norm induced by $g_{0}$. Then the $\mathfrak{t}$-valued 1-forms $\psi \lambda_{h}^{j}$ and $\psi \lambda_{h}^{j^{\prime}}$ satisfy the conditions of Theorem 4.3.

In particular, the associated pairs of metrics $g_{\psi \lambda^{j}}$ and $g_{\psi \lambda^{j^{\prime}}}$ in (i) (respectively $g_{\psi \lambda_{h}^{j}}$ and $g_{\psi \lambda_{h}^{j^{\prime}}}$ in (ii)) are isophasal with respect to $g_{0}$; if $\left(X, g_{0}\right)$ is asymptotically hyperbolic, then they are also isopolar.

Proof. (i) The assumption on the $H$ - and $T$-orbits implies that $\psi \lambda^{j}$ and $\psi \lambda^{j^{\prime}}$ are horizontal. Obviously, they are also $T$-invariant: Note that $\psi$ is so by assumption, and the vectorfields $j_{Z}^{*}$ are $T$-invariant vectorfields on $X$ since the actions of $H$ and $T$ commute. Moreover, the two 1-forms are compactly supported since so is $\psi$. It remains to show that $\psi \lambda^{j}$ and $\psi \lambda^{j^{\prime}}$ are isospectral in the sense of Definition 4.1(iii). Let $\mu \in \mathfrak{t}^{*}$, and let $Z \in \mathfrak{t}$ be the dual vector with respect to the inner product on $\mathfrak{t}$. Choose $a_{Z} \in H$ such that $j_{Z}^{\prime}=\operatorname{Ad}_{a_{Z}}\left(j_{Z}\right)$. A straightforward calculation shows that the $T$-equivariant isometry $F_{\mu}:=a_{Z}$ of $\left(X, g_{0}\right)$ satisfies $\mu \circ \lambda^{j}=F_{\mu}^{*}\left(\mu \circ \lambda^{j^{\prime}}\right)$. The same remains true for $\psi \lambda^{j}$ and $\psi \lambda^{j^{\prime}}$ since $\psi$ is $H$-invariant.

(ii) First of all, it is easy to see that $\lambda_{h}^{j}$ and $\lambda_{h}^{j^{\prime}}$ are indeed smooth; in fact, if $Y$ is a vectorfield on $X$ then $\lambda_{h}^{j}(Y)$ equals

$$
v \lambda^{j}(Y)-\sum_{i=1}^{k}\left\langle Z_{1}^{*} \wedge \ldots \wedge Z_{i-1}^{*} \wedge Y \wedge Z_{i+1}^{*} \wedge \ldots \wedge Z_{k}^{*}, Z_{1}^{*} \wedge \ldots \wedge Z_{k}^{*}\right\rangle \lambda^{j}\left(Z_{i}^{*}\right) .
$$

Now $\psi \lambda_{h}^{j}$ and $\psi \lambda_{h}^{j^{\prime}}$ are horizontal by construction. The rest of the proof is the same as for (i); one has to keep in mind that the vectorfields $Z_{1}^{*}, \ldots, Z_{k}^{*}$ are $H$ - and $T$-invariant.

Remark 4.8. (i) Let $\left(X, g_{0}\right)$ be a complete connected manifold admitting an effective action of $\mathrm{O}(m+4)$ by isometries, where $m \geq 5$. Consider the subgroup $H \times T:=\mathrm{SO}(m) \times(\mathrm{SO}(2) \times \mathrm{SO}(2)) \subset \mathrm{O}(m+4)$. Then one can use the action of $H \times T$ on $X$, along with the isospectral families of maps $j: \mathfrak{t} \rightarrow \mathfrak{h}$ from 4.5(i), to obtain continuous multiparameter families of isophasal metrics $g_{\psi \lambda_{h}^{j}}$ (with varying isospectral maps $j$ ) on $X$ as applications of Theorem 4.7(ii); one only has to choose, in addition, a compactly supported $H \times T$-invariant cut-off function $\psi$ on $X$; for example, one can choose $\psi$ to be even $\mathrm{O}(m+4)$-invariant and with support in a small neighbourhood of one of the $\mathrm{O}(m+4)$-orbits. If $\left(X, g_{0}\right)$ is asymptotically hyperbolic, then the resulting metrics are also isopolar.

(ii) If, in the situation of (i), the $H$ - and T-orbits meet orthogonally in $\left(X, g_{0}\right)$, then one obtains applications of 4.7(i). For example, this is the case for the canonical action of $\mathrm{O}(n)$ on $\mathbb{R}^{n}(n=m+4)$, when $\mathbb{R}^{n}$ is endowed with any complete $\mathrm{O}(n)$-invariant metric $g_{0}$. In this case, $\psi$ can be chosen, for example, to be any radial cut-off function on $\mathbb{R}^{n}$. One obtains continuous multiparameter families of isophasal metrics $g_{\psi \lambda^{j}}$ on $\mathbb{R}^{n}$ (with varying $j$ ), each of which is a compact perturbation of some fixed $O(n)$-invariant metric. If $\left(\mathbb{R}^{n}, g_{0}\right)$ is asymptotically hyperbolic, then these metrics are also isopolar. 


\section{Remark 4.9. (Proof of the Main Theorem)}

In section 5 we will show that the isophasal/isopolar families of metrics arising from the construction in 4.8(ii) - that is, from the canonical $O(n)$-action on $\mathbb{R}^{n}$ and a radial cut-off function $\psi$ - are generically nontrivial. The genericity condition will concern only the choice of isospectral $j$-maps, but neither the choice of rotational metric $g_{0}$ nor the choice of radial cut-off function $\psi$. Thus by Remark 4.5(i) we obtain, for any complete $O(n)$-invariant metric $g_{0}$ on $R^{n}$ and any compactly supported radial cut-off function $\psi$, continuous multiparameter families of mutually nonisometric, isophasal metrics (also isopolar, if $\left(\mathbb{R}^{n}, g_{0}\right)$ is asymptotically hyperbolic) coinciding with $g_{0}$ outside the support $K$ of $\psi$. This proves Theorem 1.1.

We conclude this section by considering several special cases of 4.8(ii) (including classes of metrics which are not asymptotically hyperbolic):

Remark 4.10. (i) Let $(r, \omega) \in \mathbb{R}_{+} \times S^{n-1}$ denote polar coordinates on $\mathbb{R}^{n}$. Let $h$ denote the round metric on $S^{n-1}$. Some canonical choices for $\left(\mathbb{R}^{n}, g_{0}\right)$ are:

$$
\begin{array}{lll}
\text { Hyperbolic space: } & g_{0(r, \omega)}=r^{2} h+\left(1+r^{2}\right)^{-1} d r^{2} \\
\text { Euclidean space: } & g_{0(r, \omega)}=r^{2} h+d r^{2} \\
\text { Paraboloid: } & g_{0(r, \omega)}=r^{2} h+\left(1+r^{2}\right) d r^{2} \\
\text { Hyperboloid: } & g_{0(r, \omega)}=r^{2} h+\left(1+\frac{1}{1+r^{2}}\right) d r^{2}
\end{array}
$$

Thus for each of these metrics $g_{0}$ we have continuous families of isophasal metrics $g_{\psi \lambda^{j}}$ on $\mathbb{R}^{n}$ which agree with $g_{0}$ outside a compact $\mathrm{O}(n)$-invariant region. In the first example (hyperbolic space), these metris are also isopolar.

(ii) After normalizing the radial coordinate, a general $\mathrm{O}(n)$-invariant, complete metric $g_{0}$ on $\mathbb{R}^{n}$ is given by

$$
g_{0(r, \omega)}=f(r) h+d r^{2},
$$

where $f$ is the restriction to $\mathbb{R}_{+}$of any smooth, even, positive function satisfying $f(r) \sim r^{2}$ for $r \downarrow 0$.

- If we choose $f(r)$ to become constant for large $r$, then $\left(\mathbb{R}^{n}, g_{0}\right)$ has a cylindrical end. If, moreover, we choose $f(r)=r^{2}$ for all $r$ in some initial interval $(0, a)$ and choose $\psi$ to have compact support away from 0 , then $\left(\mathbb{R}^{n}, g_{0}\right)$ as well as each $\left(\mathbb{R}^{n}, g_{\psi \lambda^{j}}\right)$ from an isophasal family as above will be isometric to a flat ball glued onto a standard cylinder with a compact transition region.

- If, instead, we choose $f(r)=r^{-2}$ for $r$ large, then $\left(\mathbb{R}^{n}, g_{0}\right)$ as well as the isophasal manifolds $\left(\mathbb{R}^{n}, g_{\psi \lambda^{j}}\right)$ have a cuspidal end.

\section{NON-ISOMETRY}

In this section we show that the continuous families of isophasal metrics on $\mathbb{R}^{n}$ discussed in Remark 4.8(ii) are generically nontrivial.

Let $H \times T=\mathrm{SO}(m) \times(\mathrm{SO}(2) \times \mathrm{SO}(2)) \subset \mathrm{O}(m+4)$ with $m \geq 5$. Let $\mathfrak{t} \cong \mathbb{R}^{2}$ be the Lie algebra of $T$, endowed with the standard Euclidean metric, and let $\mathfrak{h}=\mathfrak{s o}(m)$. Consider the standard action of $\mathrm{O}(n)$ on $\mathbb{R}^{n}$, where $n:=m+4$, and the induced action of $H \times T$. Let $g_{0}$ be any complete $\mathrm{O}(n)$-invariant metric on $\mathbb{R}^{n}$, and let $0 \neq \psi \in \mathcal{C}^{\infty}\left(\mathbb{R}^{n}\right)$ be a smooth, radial, compactly supported function on $\mathbb{R}^{n}$. For any linear map $j: \mathfrak{t} \rightarrow \mathfrak{h}$, let $\lambda^{j}$ be the associated 1 -form on $\mathbb{R}^{n}$ and consider the Riemannian metric $g_{\psi \lambda^{j}}$ on $\mathbb{R}^{n}$ (recall Remark 4.8(ii)). 
Theorem 5.1. Suppose that $j, j^{\prime}: \mathfrak{t} \rightarrow \mathfrak{h}$ are nonequivalent in the sense of Definition $4.4(i i)$, and that $j^{\prime}$ is generic in the sense that $j^{\prime}(\mathfrak{t})$ has trivial centralizer in $\mathfrak{h}$. Then the Riemannian manifolds $\left(\mathbb{R}^{n}, g_{\psi \lambda^{j}}\right)$ and $\left(\mathbb{R}^{n}, g_{\psi \lambda^{j^{\prime}}}\right)$ constructed as in Remark 4.8(ii) are nonisometric.

This theorem, applied to the continuous families of pairwise isospectral and nonequivalent maps $j_{t}$ from Remark 4.5(i), shows that the associated isophasal Riemannian manifolds $\left(\mathbb{R}^{n}, g_{\psi \lambda^{j_{t}}}\right)$ are nonisometric provided that all $j_{t}(\mathfrak{t})$ have trivial centralizer in $\mathfrak{h}$. But this condition is indeed generically satisfied.

To prove Theorem 5.1, we first need the following auxiliary result.

Lemma 5.2. Suppose that $j(\mathfrak{t})$ has trivial centralizer in $\mathfrak{h}$. Then $T$ is a maximal torus in $\operatorname{Isom}\left(\mathbb{R}^{n}, g_{\psi \lambda^{j}}\right)$.

Proof of Lemma 5.2. Let $F_{t}$ be a 1-parameter family of isometries of $\left(\mathbb{R}^{n}, g_{\psi \lambda^{j}}\right)$ commuting with $T$. Then the $F_{t}$ are $T$-equivariant and preserve the $g_{\psi \lambda^{j}}$-horizontal distribution on the union $\widehat{\mathbb{R}}^{n}$ of all principal $T$-orbits. Denoting by $\omega_{\psi \lambda^{j}}$ the connection form on the principal $T$-bundle $\widehat{\mathbb{R}}^{n}$ whose kernel is the $g_{\psi \lambda^{j}}$-horizontal distribution, it follows that the $F_{t}$ preserve $\omega_{\psi \lambda^{j}}=\omega_{0}+\psi \lambda^{j}$ and consequently also preserve $d \omega_{\psi \lambda^{j}}=d \omega_{0}+d\left(\psi \lambda^{j}\right)=d\left(\psi \lambda^{j}\right)$; note that $d \omega_{0}=0$ here since the $g_{0}$-horizontal distribution is integrable.

The support of $d\left(\psi \lambda^{j}\right)$ is exactly the support of $\psi$. In fact, for $x \in \mathbb{R}^{n}$ with $x \notin\{0\} \times \mathbb{R}^{4}$ and $\psi(x) \neq 0$ one can find $Z \in \mathfrak{t}$ and $X, Y \in T_{x} \mathbb{R}^{n} \cong \mathbb{R}^{n}$ tangent to the $\mathrm{O}(m)$-orbit through $x$ such that $\psi(x) \cdot 2 g_{0}\left(j_{Z} X, Y\right) \neq 0$ (using the genericity condition on $j$ and $m \geq 5>4$ ); note that this is, for vectors $X, Y$ as chosen, the same as $\left\langle d\left(\psi \lambda^{j}\right)_{x}(X, Y), Z\right\rangle$, by the $\mathrm{O}(n)$-invariance of $g_{0}$ and $\psi$ and the skewsymmetry of $j_{Z}$. We have now shown that the intersection of the support of $\psi$ with the open dense set $\mathbb{R}^{n} \backslash\left(\{0\} \times \mathbb{R}^{4}\right)$ is contained in the support of $d\left(\psi \lambda^{j}\right)$; this implies that the support of $\psi$ is contained in the support of $d\left(\psi \lambda^{j}\right)$. The converse is obvious.

Therefore, the $F_{t}$ induce a 1-parameter family of isometries $\bar{F}_{t}$ on $\left(\widehat{\mathbb{R}}^{n} / T, g_{0}^{T}\right)$ (recall that $g_{0}^{T}=g_{\psi \lambda^{j}}^{T}$ ) which preserve the support of the induced function $\bar{\psi}$ on $\widehat{\mathbb{R}}^{n} / T$. We identify $\widehat{\mathbb{R}}^{n} / T$ with the open "quadrant"

$$
Q=\left\{(p, a, b) \mid p \in \mathbb{R}^{m}, a, b>0\right\} \subset \mathbb{R}^{m+2} .
$$

Noting that $g_{0}^{T}$ is the restriction to $Q$ of an $\mathrm{O}(m+2)$-invariant metric on $\mathbb{R}^{m+2}$, and that $\operatorname{supp} \bar{\psi}$ is the intersection of $Q$ with some compact $\mathrm{O}(m+2)$-invariant set, it follows that $\bar{F}_{t}$ is of the form $\left(A_{t}, \mathrm{Id}\right)$ with a 1-parameter family $A_{t}$ in $\mathrm{SO}(m)$.

But $\bar{F}_{t}$ must preserve the form $\Omega_{\psi^{j}}$ on $\hat{\mathbb{R}}^{n} / T$ induced by the $T$-invariant, horizontal, $\mathfrak{t}$-valued 2-form $d\left(\psi \lambda^{j}\right)$. It follows for all $x \in \mathbb{R}^{n}$ in the support of $\psi$, all $X, Y \in T_{x} \mathbb{R}^{n}$ tangent to the $\mathrm{O}(m)$-orbit through $x$ and all $Z \in \mathfrak{t}$ :

$$
\begin{aligned}
\psi\left(A_{t} x\right) \cdot 2\left(g_{0}\right)_{A_{t} x}\left(j_{Z} A_{t} X, A_{t} Y\right) & =\left\langle\left(F_{t}^{*} d\left(\psi \lambda^{j}\right)\right)_{x}(X, Y), Z\right\rangle \\
& =\left\langle d\left(\psi \lambda^{j}\right)_{x}(X, Y), Z\right\rangle=\psi(x) \cdot 2\left(g_{0}\right)_{x}\left(j_{Z} X, Y\right)
\end{aligned}
$$

and hence $j_{Z} \equiv A_{t} j_{Z} A_{t}^{-1}$ for all $Z \in \mathfrak{t}$. From the genericity assumption on $j$ we conclude $A_{t} \equiv \mathrm{Id}$. Thus $F_{t}$ restricts to a gauge transformation on the principal $T$-bundle $\widehat{\mathbb{R}}^{n}$. On the other hand, we saw above that $F_{t}^{*} \omega_{\psi \lambda^{j}} \equiv \omega_{\psi \lambda^{j}}$. But a gauge transformation of $\widehat{\mathbb{R}}^{n}$ which preserves a connection form must be an element of $T$. Thus $F_{t} \in T$ for all $t$, as claimed. 
Proof of Theorem 5.1. Suppose there were an isometry $F:\left(\mathbb{R}^{n}, g_{\psi \lambda^{j}}\right) \rightarrow\left(\mathbb{R}^{n}, g_{\psi \lambda^{j^{\prime}}}\right)$. By 5.2, $T$ is a maximal torus in $\operatorname{Isom}\left(\mathbb{R}^{n}, g_{\psi \lambda^{j^{\prime}}}\right)$. Since all maximal tori are conjugate, we can assume (after possibly composing $F$ with an isometry of $g_{\psi \lambda^{j^{\prime}}}$ ) that conjugation by $F$ maps $T \subset \operatorname{Isom}\left(\mathbb{R}^{n}, g_{\psi \lambda^{j}}\right)$ to $T \subset \operatorname{Isom}\left(\mathbb{R}^{n}, g_{\psi \lambda^{j^{\prime}}}\right)$. Then $F$ maps $T$-orbits to $T$-orbits, and, letting $\tau$ denote the automorphism of $\mathfrak{t}$ induced by conjugation by $F$, we have $F_{*}\left(Z^{*}\right)=\tau(Z)^{*}$ for all $Z \in \mathfrak{t}$.

Recall that $g_{\psi \lambda^{j}}, g_{\psi \lambda^{j^{j}}}$, and $g_{0}$ are all the same when restricted to the $T$ orbits in $\mathbb{R}^{n}$. So all principal $T$-orbits are rectangular tori whose shortest closed geodesics are integral curves parametrized on $[0,2 \pi]$ of the vectorfields $\pm Z_{1}^{*}$ or $\pm Z_{2}^{*}$, where $\left\{Z_{1}, Z_{2}\right\}$ denotes the standard basis of $\mathfrak{t}$. It follows that $\tau$ preserves the set $\left\{ \pm Z_{1}, \pm Z_{2}\right\} ;$ in particular, $\tau \in \mathrm{O}(\mathfrak{t})$.

Since $F$ maps the $g_{\psi \lambda^{j}}$-horizontal distribution on $\hat{\mathbb{R}}^{n}$ to the $g_{\psi \lambda^{j^{\prime}}}$-horizontal distribution, we have $F^{*} \omega_{\psi \lambda^{j^{\prime}}}=\tau \circ \omega_{\psi \lambda^{j}}$ for the associated connection forms; in particular, $F^{*} d\left(\psi \lambda^{j^{\prime}}\right)=F^{*} d \omega_{\psi \lambda^{j^{\prime}}}=\tau \circ d \omega_{\psi \lambda^{j}}=\tau \circ d\left(\psi \lambda^{j}\right)$. Using the facts that $\operatorname{supp} \psi=\operatorname{supp}\left(d\left(\psi \lambda^{j}\right)\right)=\operatorname{supp}\left(d\left(\psi \lambda^{j^{\prime}}\right)\right)$ is $\mathrm{O}(n)$-invariant and that $g_{0}^{T}$ is the restriction of an $\mathrm{O}(m+2)$-invariant metric to the open quadrant $Q$ representing $\hat{\mathbb{R}}^{n} / T$, we now see, just as in the proof of Lemma 5.2 , that $\bar{F}$ is of the form $(A, \sigma) \in$ $\mathrm{O}(m) \times \mathrm{O}(2)$ on the quadrant $Q \subset \mathbb{R}^{m+2}$ (where $\sigma$ is either Id or interchanges the two coordinates of $\mathbb{R}^{2}$ ).

By a similar calculation as in the proof of Lemma 5.2 and using $\tau \in \mathrm{O}(\mathfrak{t})$, we conclude that $j_{Z}^{\prime}=A j_{\tau^{-1}(Z)} A^{-1}$ for all $Z \in \mathfrak{t}$, contradicting the nonequivalence assumption.

\section{REFERENCES}

[1] Bérard, P. Transplantation et isospectralité. Math. Ann. 292 (1992), 547-560.

[2] Brooks, R., Davidovich, O. Isoscattering on Surfaces. J. Geom. Anal. 13 (2003), no. 1, 39-53.

[3] Brooks, R., Gornet, Ruth; Perry, Peter. Isoscattering Schottky manifolds. Geom. Funct. Anal. 10 (2000), no. 2, 307-326.

[4] Brooks, Robert; Perry, P. Isophasal scattering manifolds in two dimensions. Comm. Math. Phys. 223 (2001), no. 3, 465-474.

[5] Carron, G. Déterminant relatif et la fonction Xi. (French) [Relative determinant and the Xi-function] Amer. J. Math. 124 (2002), no. 2, 307-352.

[6] Christiansen, T.; Zworski, M. Resonance wave expansions: two hyperbolic examples. Comm. Math. Phys. 212 (2000), no. 2, 323-336.

[7] Croke, C.; Sharafutdinov, V. Spectral rigidity of a compact negatively curved manifold. Topology 37 (1998), no. 6, 1265-1273.

[8] Duistermaat, H., Guillemin, V. The spectrum of positive elliptic operators and periodic bicharacteristics. Invent. Math. 29 (1975), no. 1, 39-79.

[9] Gordon, C. Isospectral deformations of metrics on spheres. Invent. Math. 145 (2001), 317331.

[10] Gordon, C. Isospectral closed Riemannian manifolds which are not locally isometric. II. Geometry of the spectrum (Seattle, WA, 1993), 121-131, Contemp. Math., 173, Amer. Math. Soc., Providence, RI, 1994.

[11] Gordon, C; Perry, P. Continuous families of isophasal scattering manifolds. Math. Res. Lett., to appear.

[12] Gordon, C., Szabo, Z. Isospectral deformations of negatively curved Riemannian manifolds with boundary which are not locally isometric. Duke Math. J. 113 (2002), no. 2, 355-383.

[13] Gordon, C., Wilson, E. Continuous families of isospectral Riemannian manifolds which are not locally isometric. J. Diff. Geom. 47 (1997), 504-529.

[14] Guillarmou, C. Meromorphic properties of the resolvent on asymptotically hyperbolic manifolds. Preprint, Arxiv:Math.SP/0311424. 
[15] Guillarmou, C. Resonances and scattering poles on asymptotically hyperbolic manifolds. Submitted to Int. Math. Res. Notes.

[16] Guillemin, V., Kazhdan, D. Some inverse spectral results for negatively curved 2-manifolds. Topology 19 (1980), no. 3, 301-312.

[17] Guillopé, L., Zworski, M. Upper bounds on the number of resonances for non-compact Riemann surfaces. J. Funct. Anal. 129 (1995), 364-389.

[18] Guillopé, L., Zworski, M. Polynomial bounds on the number of resonances for some complete spaces of constant curvature. Asymptotic Anal. 11 (1995), 1-22.

[19] Guillopé, L., Zworski, M. Scattering asymptotics for Riemann surfaces. Ann. Math. 145 (1997), 597-660.

[20] Joshi, M., Sá Barreto, A. Inverse scattering on asymptotically hyperbolic manifolds. Acta Math. 184 (2000), no. 1, 41-86.

[21] Kato, T. Perturbation Theory for Linear Operators. Berlin, New York: Springer-Verlag, 1966.

[22] Mazzeo, R. Unique continuation at infinity and embedded eigenvalues for asymptotically hyperbolic manifolds. American J. Math. 113 (1991), 25-56.

[23] Mazzeo, R; Melrose, R. Meromorphic extension of the resolvent on complete spaces with asymptotically constant negative curvature. J. Funct. Anal. 75 (1987), 260-310.

[24] Melrose, R. Geometric Scattering Theory. New York, Melbourne: Cambridge University Press, 1995.

[25] Min-Oo, M. Spectral rigidity for manifolds with negative curvature operator. Nonlinear problems in geometry (Mobile, Ala., 1985), 99-103, Contemp. Math., 51, Amer. Math. Soc., Providence, RI, 1986.

[26] Müller, W. Relative zeta functions, relative determinants and scattering theory. Comm. Math. Phys. 192 (1998), no. 2, 309-347.

[27] Proctor, E. Isospectral metrics on classical compact simple Lie groups. Ph.D. thesis, Dartmouth College, 2003.

[28] Reed, M., Simon, B. Methods of Modern Mathematical Physics, II. Fourier Analysis, ,SelfAdjointness. New York, Academic Press, 1975.

[29] Reed, M., Simon, B. Methods of Modern Mathematical Physics, III. Scattering Theory. New York, Academic Press, 1979.

[30] Schueth, D. Continuous families of isospectral metrics on simply connected manifolds. Ann. of Math. (2) 149 (1999), no. 1, 287-308.

[31] Schueth, D. Isospectral manifolds with different local geometries. J. reine angew. Math. 534 (2001), 41-94.

[32] Schueth, D. Isospectral metrics on five-dimensional spheres. J. Differential Geom. 58 (2001), no. $1,87-111$.

[33] Tang, S.-H., Zworski, M. Resonance expansions of scattered waves. Comm. Pure Appl. Math. 53 (2000), no. 10, 1305-1334.

[34] Yafaev, D. R. Mathematical scattering theory. General theory. Translated from the Russian by J. R. Schulenberger. Translations of Mathematical Monographs, 105. American Mathematical Society, Providence, RI, 1992.

[35] Zelditch, S. Isospectrality in the FIO category. J. Differential Geom. 35 (1992), no. 3, 689710.

[36] Zelditch, S. Kuznecov sum formulae and Szegö limit formulas on manifolds. Comm. P. D. E. 17 (1992), 221-260.

[37] Zworski, M. Resonance expansions in wave propagation. Séminaire: Équations aux Dérivées Partielles, 1999-2000, Exp. No. XXII, 11 pp., Sémin. Équ. Dériv. Partielles, École Polytech., Palaiseau, 2000.

(Perry) Department of Mathematics, University of Kentucky, Lexington, Kentucky 40506-0027, U. S. A.

E-mail address: perry@ms.uky.edu

(Schueth) Inst. f. Math., HU Berlin, 10099 Berlin, Germany

E-mail address: schueth@math.hu-berlin.de 